$\begin{array}{cl}\begin{array}{c}\text { Revue } \\ \text { de lhistoire }\end{array} & \text { Revue de l'histoire des religions } \\ \text { des religions } & 4 \mid 2005 \\ & \begin{array}{l}\text { Lieux de culte, lieux saints dans le judaïsme, le } \\ \text { christianisme et l'islam }\end{array}\end{array}$

\title{
La mosquée et le sanctuaire Sainteté des lieux en Islam
}

The Mosque and the Sanctuary. Holy Character of Places in Islam

\section{Oleg Grabar}

\section{(2) OpenEdition \\ Journals}

Édition électronique

URL : http://journals.openedition.org/rhr/4225

DOI : $10.4000 /$ rhr.4225

ISSN : 2105-2573

Éditeur

Armand Colin

Édition imprimée

Date de publication : 1 octobre 2005

Pagination : 481-489

ISBN : 2200-92087-3

ISSN : 0035-1423

Référence électronique

Oleg Grabar, "La mosquée et le sanctuaire Sainteté des lieux en Islam », Revue de l'histoire des religions [En ligne], 4 | 2005, mis en ligne le 15 janvier 2010, consulté le 19 avril 2019. URL : http:// journals.openedition.org/rhr/4225; DOI : 10.4000/rhr.4225 


\section{La mosquée et le sanctuaire Sainteté des lieux en Islam}

La présente contribution propose, à titre préliminaire, de considérer l'idée du sacré dans le monde de l'Islam à partir de l'architecture et de distinguer trois catégories de monuments religieux : 1) les grands sanctuaires panislamiques (comme La Mecque, Médine et Jérusalem) dont le caractère sacré est plus ou moins constant ; 2) la mosquée, aux valeurs sacrées variables selon le moment; 3) les lieux saints (mashahid), qui, quelles qu'en soient les formes, reflètent les besoins des fidèles dans leurs espaces aussi bien que dans les pratiques que l'on y décèle.

\section{The Mosque and the Sanctuary. Holy Character of Places in Islam}

As a preliminary, we propose to consider the concept of holiness in the Islamic world starting with architecture, and distinguishing three categories of religious buildings : 1) Large pan islamic sanctuaries (such as Mecca, Medina, and Jerusalem), the sacred character of which remains more or less steady ;2) Mosques the sanctity of which varies according to time ; 3) Holy places (mashahid) whatever shape they may assume, answering the needs of the faithfuls in the space offered as well as in the practices available. 
Les savants ont deux façons de connaître et de comprendre leurs domaines de recherche. L'une consiste à considérer chaque sujet comme indépendant ; ce dernier crée ses propres termes épistémologiques et ses propres formules d'expression. L'autre façon est de rechercher et de trouver des formules générales, plus ou moins universelles, dans lesquelles on pourrait caser le phénomène particulier que l'on étudie. « Religieux », « sacré », « saint », « sanctuaire » font partie de la catégorie de termes généraux qui impliquent des pratiques et des paradigmes universels et pourtant qui soulèvent toutes sortes de problèmes lorsqu' on cherche à les employer dans des situations historiques ou des contextes culturels concrets différents de ceux qui les ont créés ou utilisés en premier. Et, pourtant, nous ne pouvons communiquer les uns avec les autres que si nous disposons d'un vocabulaire et d'une syntaxe communs. Le dévergondage des mots dans le langage des médias et des ordinateurs est là pour montrer l'importance de donner aux mots des sens clairs et précis, mais aussi l'utilité douteuse d'une telle démarche, car les transformations de sens n'en restent pas moins vivaces et acceptées par le grand public. Je vais, cependant, esquisser une approche possible à ce problème tout en soulignant que mes remarques ne sont que des observations demandant à être discutées, voire contredites.

Ces remarques préliminaires m'ont été inspirées par quelques années de recherche discontinue et intermittente sur le sujet du «sacré » dans le monde de l'Islam, et plus particulièrement dans son architecture et dans les choses, les objets, les motifs du monde visuel créé par l'Islam. Mes investigations ont commencé d'une manière bien simple avec l'écriture arabe dont la transformation en calligraphie, fréquente et - semble-t-il - déjà apparente au premier siècle de l'hégire, a été interprétée par certains savants orientalistes comme Annemarie Schimmel ${ }^{1}$, ou bien des critiques et historiens de l'art comme Erica Dodd, Sayyid Husain Nasr ou André Clevenot ${ }^{2}$, ou

1. Annemarie Schimmel, Calligraphy in Islamic Culture (New York, 1983).

2. Erica Dodd, "The Image and the Word", Berytus, 1969; Sayyid Husayn Nasr, Islamic Art and Spirituality (Albany, N. Y., 1987) ; Dominique Clevenot, Esthétique du Voile : essai sur l'Art arabo-islamique (Paris 1994), entre beaucoup d'autres exemples. 
bien des artistes comme M. Sijilmassi, Dia Azzaoui, ou Muhammad Zakariya, et bien d'autres encore, comme étant l'expression du sacré en Islam. Le Verbe aurait pris le relais de l'Image. Et il est en effet exact que des transformations de l'écriture existent, surtout dans le monde iranien et irano-centrique, mais également sous les Ottomans, dans le monde sunnite aussi bien que parmi les shi'ites, qui deviennent, comme les icônes chrétiennes ou les objets pieux bouddhistes ou hindous, des images plutôt que des textes qui, par leur caractère, incitent à entrer en relations directes avec elles, qui excluent leur contexte et inclinent à la ferveur religieuse ${ }^{3}$.

Intellectuellement, il m'est facile de proposer des interprétations spirituelles de ces œuvres d'art, mais le raisonnement qui me conduit à ces interprétations n'est pas un raisonnement religieux. Il n'est pas l'expression de ma foi, si tant est que j'en aie une, ni de ma connaissance directe de l'attitude d'un musulman devant ces œuvres ou de l'artisan ou de l'artiste qui les créa. Ce que je fais en proposant ces explications, c'est d'identifier dans les formes des textes associés à la foi et d'en déduire une pratique pieuse basée non pas directement sur l'expérience musulmane mais sur celle d'un conglomérat de religions, parmi lesquelles dominent le christianisme et l'hindouisme, religions bien étudiées par les historiens. Parce que certaines formules formelles - perception immédiate de l'image tout entière, composition unifiée et non pas narrative, symétrie du dessin, centralisation de la composition, et ainsi de suite - se retrouvent couramment dans des arts au contexte religieux bien établi et analysé par Mircea Eliade, Rudolph Otto ou Roger

3. Quelques exemples : un panneau en stuc du XIVe siècle à Linjan, près d'Isfahan ; une célèbre peinture du Xv siècle avec le nom d'Ali transformé en une étonnante image géométrique ; une représentation du mot «Allah », Dieu, $\mathrm{du} \mathrm{XV}^{\mathrm{e}}$ siècle également dans la mosquée de Yazd, fait avec le texte tout entier de la première sourate du Coran ; les hilyes ottomanes, qui sont des descriptions hagiographiques du Prophète parfois arrangées sous la forme de reliques associées au Prophète ; des images plus tardives du Gujarat en Inde avec les lettres arrangées sous la forme d'un visage. On trouvera ces exemples illustrés dans Oleg Grabar, L'Ornement (Paris, 1992), fig. 25, 34, 53, 57 ; Oleg Grabar et Mika Natif, "The story of portraits of the Prophet Muhammad", Studia Islamica (à paraître). 
Caillois ${ }^{4}$, la sacralité ou la sainteté de ces œuvres semblait claire et indiscutable. Mais cette conclusion ne découlait pas de l'analyse contextuelle de l'œuvre d'art, seulement de sa coïncidence avec une théorie abstraite du sacré dans l'art. Il me semblait que je m'étais fourré entre l'enclume des mots à association pieuse et le marteau d'une vision d'habitudes pieuses universelles.

Pour essayer d'en sortir, je voudrais me tourner vers les monuments d'architecture qui ont deux avantages méthodologiques. L'un est que, du moins dans notre connaissance des arts musulmans, l'on en connaît l'histoire, les sources créatrices et les causes et modalités de leur évolution, mieux que pour les objets ou la décoration. L'autre est que beaucoup de ces monuments sont encore utilisés ; on peut décrire ou reconstituer les attitudes et les sentiments qui s'attachent à leurs activités, même si je ne sais pas s'il existe des études psychologiques ou sociologiques sur ce que le croyant musulman fait, sent ou voit dans les établissements dits religieux qui l'entourent.

D'une manière générale et peut-être simplifiée, il y a trois types de monuments d'architecture religieuse :

\section{Les grands sanctuaires panislamiques}

Déjà reconnus comme tels par Ibn Khaldun ${ }^{5}$. Ils sont trois : la Ka'bah à La Mecque, la Mosquée du Prophète à Médine et le Haram al-Sharif à Jérusalem. Chacun d'entre eux a son histoire et, sauf pour la Ka'bah, leur sainteté a rarement été continue. Ce n'est que pour le Haram al-Sharif que des études récentes ont montré les complexités du développement d'un grand complexe religieux ${ }^{6}$.

4. Rudolph Otto, Idea of the Holy (London, 1956) ; Roger Caillois, L'homme et le Sacré (Paris, 1949) ; Mircea Eliade, Le Sacré et le Profane (Paris, 1965); Paul Ricœur, Figuring the Sacred (Minneapolis, 1995), une anthologie de ses travaux et pensées sur le sujet. Ce ne sont là que quelques introductions aux théories générales sur le sacré.

5. Ibn Khaldun, Al-Muqaddimah, Discours sur l'Histoire Universelle, trad. Vincent Monteil (Beyrouth, 1967-1968), p. 553 et ss.

6. O. Grabar, The Shape of the Holy (Princeton, 1996) ; Andreas Kaplony, The Haram in Jerusalem, 324-1099: temple, Friday Mosque, area of spiritual power (Stuttgart, 2002). 
Pour La Mecque comme pour Médine, les arguments archéologiques sont presque entièrement absents et nous dépendons de mentions occasionnelles dans les chroniques, de descriptions en général tardives, et d'une histoire sacrée qui a su associer les formes des bâtiments à une histoire religieuse parfois douteuse ${ }^{7}$. Dans les trois cas la sainteté du monument s'est étendue à la ville qui l'abrite. La Mecque, Médine et Jérusalem sont, de manières d'ailleurs fort différentes, des villes saintes génératrices de valeurs spirituelles variées et créatrices de toute une industrie de souvenirs. Ce qui est également important, c'est que ces trois monuments sont très différents les uns des autres et, avec une exception partielle pour la Mosquée de Médine, n'ont pour ainsi dire jamais servi de modèle à suivre.

\section{Les mosquées}

Il y en a des milliers avec des formes et des caractéristiques fort différentes selon les lieux et les époques. Curieusement, ces formes ont été étudiées par les historiens de l'art bien plus que par les historiens ou les sociologues de la religion. Dans le contexte de cette rencontre, je me limiterai aux remarques suivantes sur la mosquée :

a) Il s'agit d'un espace séparé physiquement de ce qui l'entoure, dont l'accès peut être contrôlé selon des modalités qui ont varié dans le passé comme elles varient encore aujourd'hui. Cet espace est, la plupart du temps, réservé aux croyants pour leurs activités religieuses, mais ce n'est pas un espace dont les non-Musulmans sont exclus, malgré certaines pratiques contemporaines. L'intérieur comprend en général une cour et un espace couvert et un certain

7. Les résultats de ce genre d'enquête sont parfois contradictoires. Voir, par exemple, Jean Sauvaget, La Mosquée Omeyyade de Médine (Paris, 1947, un modèle du genre en son temps et Jeremy Johns, "The House of the Prophet and the Conception of the Mosque" in J. Johns ed., Bayt al-Maqdis II (Oxford, 1999). Le sanctuaire de La Mecque est encore à la recherche de son historien et le texte si riche en renseignements que sont les Akhbar Makkah d'al-Azraqi manque de commentateurs assidus; Ferdinand Wüstenfeld, Geschichte und Beschreibung der Stadt Mekka (Leipzig, 1858). 
nombre de points fixes et constants aux valeurs pratiques ou symboliques (parfois les deux) : minbar, mihrab, minaret, maqsura, et divers éléments de moindre importance. Aucun de ces éléments, sauf le mihrab, n'a eu une histoire continue et aucune de ces histoires n'a encore été écrite.

b) À l'opposé des trois grands sanctuaires, dont le sens et la valeur changent relativement peu au cours des siècles - même si ces valeurs peuvent acquérir une intensité particulière à certaines époques comme lors des pèlerinages -, l'intensité de la pratique musulmane dans les mosquées varie d'heure en heure et de place en place. On peut prier tout seul n'importe où et à n'importe quel moment, ou bien c'est la communauté tout entière qui prie ensemble. Cela est certes vrai des églises chrétiennes, mais il est rare que ces dernières soient également les lieux de rencontres individuelles et collectives que sont les mosquées. Les nombreuses fonctions urbaines remplies par les mosquées les rendent uniques, tout au moins jusqu'à ces dernières années.

Tout comme leurs collègues qui s'occupent des arts chrétien ou hindou, les historiens de l'art musulman peuvent montrer que la décoration des mosquées comprend dans son ornement ou bien dans ses inscriptions des messages qui donnent un sens particulier à telle mosquée ou bien qui cherchent à inspirer les croyants ${ }^{8}$. Ce que nous ne savons pas, c'est comment ces formes et les sens qu'on peut leur attribuer avaient été perçus au cours des siècles.

Il y aurait ainsi dans chaque mosquée quatre éléments constitutifs - l'espace, la décoration, les activités, le sens symbolique. Aucun de ces éléments n'est saint ou sacré en soi, mais tous peuvent être transformés en chose sainte ou sacrée par le temps ou par des événements extérieurs. Par le temps, comme dans la transformation de la grande mosquée de Fatehpur Sikri en Inde par la présence du

8. Je ne donne que l'exemple récent de la Grande Mosquée de Damas telle qu'elle a été interprétée récemment par Nasser Rabbat, "The Dialogic dimension in Umayyad Art", RES, 43 (2003) avec des notes citant entre autres les importants travaux récents de Finbarr Barry Flood, comme The Great Mosque of Damascus: studies on the makings of an Umayyad visual culture (Leiden, 2001). 
tombeau d'un homme saint ou de celle de Damas par le tombeau de saint Jean. Par des événements extérieurs, comme la décision de Lyautey d'interdire aux non-musulmans l'accès des mosquées ou bien les conflits entre Musulmans et Hindous, qui ont donné une charge religieuse à des monuments, parfois à des ruines, dont cette charge était absente.

La conclusion ou hypothèse que je propose à la réflexion générale serait donc qu'au niveau des grands établissements au public restreint que sont les mosquées, on ne peut pas parler de la sainteté des lieux parce qu'il n'y avait pas d'organisation para-ecclésiastique pour maintenir la sainteté. Des actes pieux ne sont pas garants de la sainteté de l'espace dans lesquels ils se produisent.

Je passe sur les petites mosquées, les simples masajid, les mosquées privées et les mosquées de quartier qui parfois se rapprochent de ma troisième catégorie de sanctuaires musulmans.

\section{Les lieux saints}

C'est par ce terme général et inadéquat - peut-être faudrait-il parler de «lieux de témoignage », mashahid - que je voudrais considérer les monuments les plus originaux de l'architecture religieuse musulmane et qui expriment au mieux à la fois la ferveur des masses et un attachement exclusif à des lieux précis.

Il s'agit en vaste majorité de tombeaux autour desquels on a construit des mausolées et auxquels se sont adjointes toutes sortes d'institutions à fonctions pratiques. Il y a plusieurs catégories de ces mausolées. Tout d'abord, nous avons ceux des imams shi'ites (Kerbela, Najaf en Iraq, Qum ou Mashad en Iran) qui ont souvent reçu, avec leurs coupoles dorées, un développement particulièrement spectaculaire $^{9}$. Et puis nous trouvons les fondateurs d'écoles de jurisprudence (tombeaux d'al-Hanafi à Baghdad ou d'al-Shafi'i

9. À ma connaissance, aucun de ces grands sanctuaires n'a été étudié d'une manière scientifique et nous n'en possédons que des descriptions sommaires dans des guides ou bien des références incomplètes. Voir entretemps les articles consacrés à ces villes saintes dans l'Encyclopédie de l'Islam ou bien dans l'Encyclopaedia Iranica. 
au Caire) ou de fraternités sectaires (tombeau de Shaykh Yasavi à Turkestan au Kazakhstan ou celui de Jelal al-Din Rumi à Konya), ou bien encore les grands prophètes bibliques (sanctuaire de Jonas à Ninive près de Mossoul ou bien d'Abraham à Urfa et ailleurs) ${ }^{10}$. Et enfin c'est par centaines que se comptent les qubab (coupoles ou tombes) et gunbadhs (tombes) qui du Maroc au Bangladesh rappellent la présence de personnages, souvent inconnus, qui ont joué un rôle à des moments précis, même s'ils sont oubliés, de l'histoire et qui continuent à apaiser et à aider les visiteurs croyants. Il y a même, quoique plus rarement que pour le christianisme ou le bouddhisme, des sanctuaires autour de reliques plutôt que des tombes, surtout dans le cas de descendants du Prophète. Dans certains cas, je pense au sanctuaire dit Shah-i Sinda à Samarkande et à certains des grands cimetières du Caire, de vraies nécropoles se créent autour du tombeau d'un saint homme.

Deux caractéristiques générales peuvent être appliquées à ces lieux de témoignage. Tout d'abord, ce sont des lieux qui agissent, qui vivent, qui répondent aux demandes des fidèles, auxquels on apporte des dons, autour desquels on se rencontre. Par la présence des morts au milieu des vivants, ils démontrent en fait la vie éternelle possible pour tous.

L'autre caractéristique est que ce sont des lieux autour desquels, bien plus que dans les mosquées, se sont cristallisées trois des quatre fonctions religieuses typiques. Ce sont des lieux de mémoire avec des souvenirs rappelés collectivement ou individuellement. Ce sont des lieux de pèlerinage, parfois panislamiques, bien plus souvent purement locaux. Ce sont des lieux de réunion pour un vaste ensemble d'activités possibles, y compris des fonctions éducatrices ou de santé publique. Seule la fonction rituelle est peu claire, dans le sens qu'il ne semble pas que des rites communs aient été élaborés pour ces mashahid. À la différence du christianisme, des religions de l'Inde ou du bouddhisme, l'Islam n'a pas eu de grand programme liturgique. En dehors du pèlerinage à La Mecque, la seule exception possible serait autour des grands sanctuaires shi'ites d'Iraq et d'Iran

10. La bibliographie sur ces monuments est énorme, surtout sur les madrasas urbaines avec leurs tombeaux. 
et de leur extension en Inde, surtout lors des fêtes célébrant le souvenir de la mort d'Ali et de son fils.

Certains de ces lieux de mémoire avaient eu des fonctions religieuses avant l'Islam, mais je suis de moins en moins persuadé par l'hypothèse de la pérennité des lieux saints qui avait été chère à la première génération des islamologues. Leur histoire est trop souvent locale et individuelle. Même si les ressorts psychologiques de ces lieux - la peur de la mort et l'espoir d'une vie éternelle - sont universels, leur création et les formes qu'ils prirent sont particuliers à l'Islam.

Une dernière remarque sur ces lieux de témoignage. À la différence de La Mecque, de Médine et de Jérusalem, ils me semblent rarement accompagnés d'objets ou d'images et de symboles qui refléteraient le caractère particulier de chaque lieu. Il y a quelques exceptions dans le monde shi'ite, mais elles sont restées, pour la plupart, à un stade folklorique. Et il serait juste d'ajouter que, dans une mesure extrêmement difficile à déterminer, les activités et les croyances associées à ces lieux se rapprochent du monde de la magie, un tout autre domaine d'études ${ }^{11}$.

grabar@ias.edu

11. Ce texte est plus ou moins celui qui fut présenté à la réunion de L'Arbresle. Les images ont été remplacées par des références bibliographiques. 
